\title{
"Exuberant" Neuronal Growth After Brain Damage in Adult Rats: The Essential Role of Behavioral Experience
}

\author{
Timothy Schallert and Theresa A. Jones \\ Department of Psychology and Institute for Neuroscience \\ University of Texas at Austin, Austin, Texas 78712, USA
}

\section{KEY WORDS}

plasticity, dendritic branching, recovery of function, motor cortex, forelimb asymmetries, Golgi-Cox, pyramidal neuron, use-dependency

\section{INTRODUCTION}

Traditional investigations of the neural basis of recovery of function after brain damage frequently involve an analysis of behavioral recovery and of potentially related neural changes that might mediate the recovery. We recently have taken a quite different approach. Instead of assuming that the neural changes mediate (or permit) the behavioral, we showed that the opposite can be true: that behavioral events are absolutely necessary for the neural changes to take place $/ 7-9 /$. These studies, reviewed below, have led us further to hypothesize that brain damage can sensitize, or prepare, remaining neural tissue through growth promoting and pruning-like mechanisms so that in the presence of appropriate behavioral pressure, the tissue can be drastically altered to compensate for lost function. In this scheme, (a) the neural events not only require behavioral experience (usedependency), but (b) even with extensive behavioral experience, the neural changes may not be initiated unless there is also brain damage, and (c) there may be a sensitive period after the brain damage occurs during which the behavioral experience can maximally modify the neural events.

Reprint address:

T. Schallert

Department of Psychology

Mezes Hall $330 \mathrm{Z}$

University of Texas at Austin

Austin, Texas 78712, USA

\section{STRUCTURAL EVENTS}

Following unilateral injury to the forelimb representation area (FL) of the sensorimotor cortex in rats, there is a substantial transient expansion of the size of the identical cortical area of the opposite hemisphere $/ 6,8 /$. The expansion appeared to be greatest in regions containing large pyramidal neurons in layer $\mathrm{V}$ and no significant expansion was observed in a sample of other cortical regions outside the homotopic forelimb area. Layer $\mathrm{V}$ pyramidal neurons are the major output neurons of the cerebral cortex. These neurons communicate with subcortical structures and motor neurons of the spinal cord and they receive intracortical, subcortical, and transcortical input. Because cortical thickening or volume increases have been shown to reflect enhanced dendritic arborization of neurons $/ 11 /$, we examined the dendritic arbors of layer $\mathrm{V}$ pyramidal neurons in FL using Golgi Cox procedures $/ 7,8 /$.

We found a time-dependent dramatic increase in the complexity and extent of dendritic arborization that was greatest at 18 days postoperative, followed by a partial reduction in arborization that did not return to control levels even up to 120 days (Fig. 1). Increases in dendritic branching have been associated with increased numbers of synapses $/ 18,24 /$, which could possibly provide enhanced information processing and motor control capacities. The pruning of arbors is reminiscent of eliminative events associated with the plasticity of the developing brain $/ 2,14,17,19,21,22 /$ and is in line with suggestions that neural events occurring after brain damage may parallel certain mechanisms found in ontogeny $/ 23 /$. 


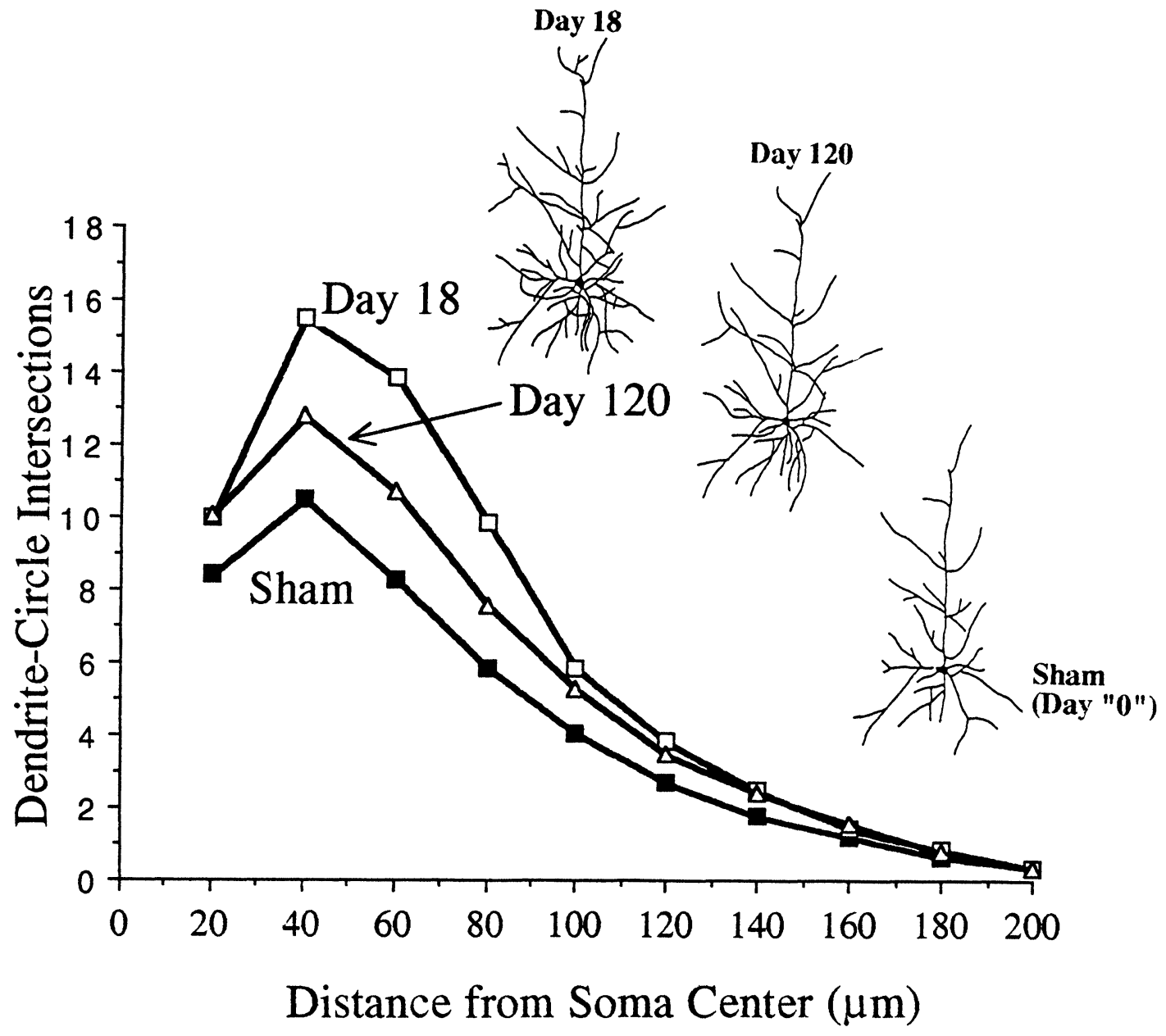

Fig. 1: Arborization of layer $\mathrm{V}$ pyramidal neuron basilar dendrites as measured using the concentric circle method. Concentric circles spaced at $20 \mu \mathrm{m}$ intervals are superimposed over camera-lucida drawings (450x) of the neurons, and the number of intersections between dendritic processes and the circles are counted to give an index of the spatial extent and size of the dendritic trees. Arborization increases were maximum at 18 days after the lesion, most notably within an $80 \mu$ radius from the soma center. At 120 days, the arbors were only modestly increased relative to shams. In addition to the increase in the spatial size of the arbors, the number of dendritic branches were increased following the lesion, following the same pattern of overgrowth and partial elimination (see Jones and Schallert / $9 /$ for details, including other time points). Pyramidal neurons on the right are schematic drawings and are roughly representative of dendritic branch number and arbor size.

\section{BEHAVIOR-ANATOMY RELATIONSHIP}

The overgrowth of dendrites appeared to correspond closely in time to postoperative changes in the use of the forelimbs for support in standing, pushing off during rearing movements, and for movement along the walls of the cage $/ 7,8 /$. That is, following the unilateral lesion, there was disuse of the forelimb contralateral to the damage and over- dependence on the ipsilateral forelimb. The ipsilateral limb was not overactive relative to control groups - rather, because the contralateral forelimb was impaired, the animals relied upon the ipsilateral forelimb to maintain balance and to prevent the contralateral forelimb from falling through grid openings or slipping down walls during vertical exploration. With time, the 
asymmetry of limb use abated significantly, which corresponded to the pruning phase. Because the forelimb postural-motor behaviors were so frequently part of their everyday movements, and because the overbranching and pruning of Layer $\mathrm{V}$ neurons occurred in FL sensorimotor cortex representing the non-impaired limb, it seemed reasonable that the arborization enhancement, and possibly the pruning as well, might be driven by behavioral experience. This idea derived in part from the work of Greenough and colleagues, who have shown that experience and skilled use of forelimbs can increase dendritic arborization in subpopulations of Layer II, III and V neurons $13,27 /$, albeit less dramatically than that observed in our brain damaged animals $/ 8 /$.

\section{OVERGROWTH/USE-DEPENDENCY LINK ESTABLISHED}

To determine whether arbor expansion in the intact cortex was driven by over-reliance on the non-impaired forelimb we designed lightweight vests, made of plaster of paris casting material, that restricted use of either the non-impaired or impaired forelimb throughout the period of neural overgrowth $15,9 /$. An additional group of rats was left uncasted and another was fitted with two-holed control casts which allowed free use of either forelimb. All animals adapted readily to the casts. The animals were well handled and were cleaned and brushed at least twice daily. A cotton probe was used to clean and stimulate the regions under the cast. Enough space was left under the one-sleeved casts to allow minor movements since the purpose of these casts was only to limit use of one forelimb for postural supporting and ambulatory behaviors. When the casts were removed, the animals were immediately able to use the restricted forelimb for locomotor or other behaviors.

The major result was that restricting use of the non-impaired limb completely prevented the dendritic overgrowth that otherwise would have occurred in the intact FL sensorimotor cortex (Fig. 2) $/ 5,9 /$. In contrast, it was no surprise that control casts or casts which simply restricted use of the impaired forelimb (leaving the non-impaired limb free to engage in compensatory postural supporting behaviors, as though no cast had been applied), had no diminishing effect on dendritic overgrowth. These results were consistent with the view that forelimb experience played an essential role in the dendritic overgrowth.

\section{LESION-DEPENDENCY ESTABLISHED}

On the other hand, forced use of one forelimb in sham-operated animals having no brain damage failed to promote reliable dendritic overgrowth. These animals were fitted with a cast that prevented use of one forelimb for 15 days; nevertheless, no significant increase in arborization occurred in Layer V neurons of FL sensorimotor cortex contralateral to the overused forelimb /9/. Thus, the dramatic arborization effect may require brain damage.

We also found that non-cortical unilateral brain injury, even when it produces severe and chronic disuse of the contralateral forelimb, is not sufficient to permit the enhanced dendritic arborization, at least not in FL cortex. For example, a group of animals sustained severe unilateral depletion of dopamine in the nigrostriatal system and was sacrificed 15-18 days later $/ 9,12 /$. There was no increase in arbor size or complexity in the cortex contralateral to the compensating forelimb (despite limb use asymmetries that were even greater than that observed after unilateral sensorimotor cortex lesions).

Likewise, no detectable interhemispheric asymmetries in dendritic arborization or spine density of Layer V neurons in FL cortex were found in rats after unilateral pyramidal tract lesions $/ 25 /$. These animals showed extreme disuse of one forelimb during the first few postoperative weeks, and even at the time of sacrifice (6 months), normal limb use had not been restored. It appears, therefore, that degenerative or related signals within the cortex $/ 10,12,14,15 /$ may be necessary for the structural events to occur there $/ 9 /$. 
Lesion-No Cast neither forelimb restricted

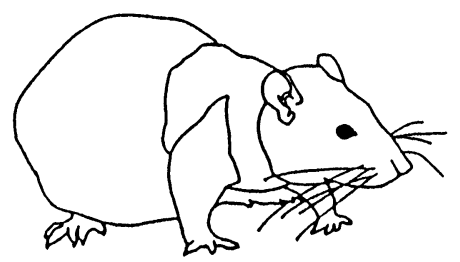

Lesion+Contra Cast impaired forelimb restricted
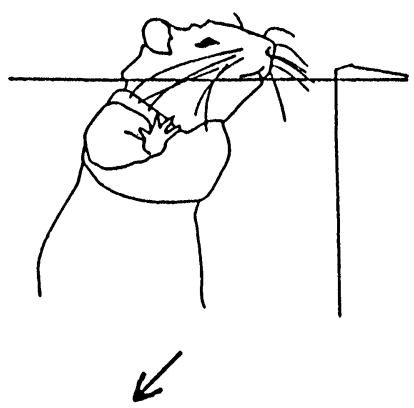

Increased dendritic arborization in cortex opposite the lesion
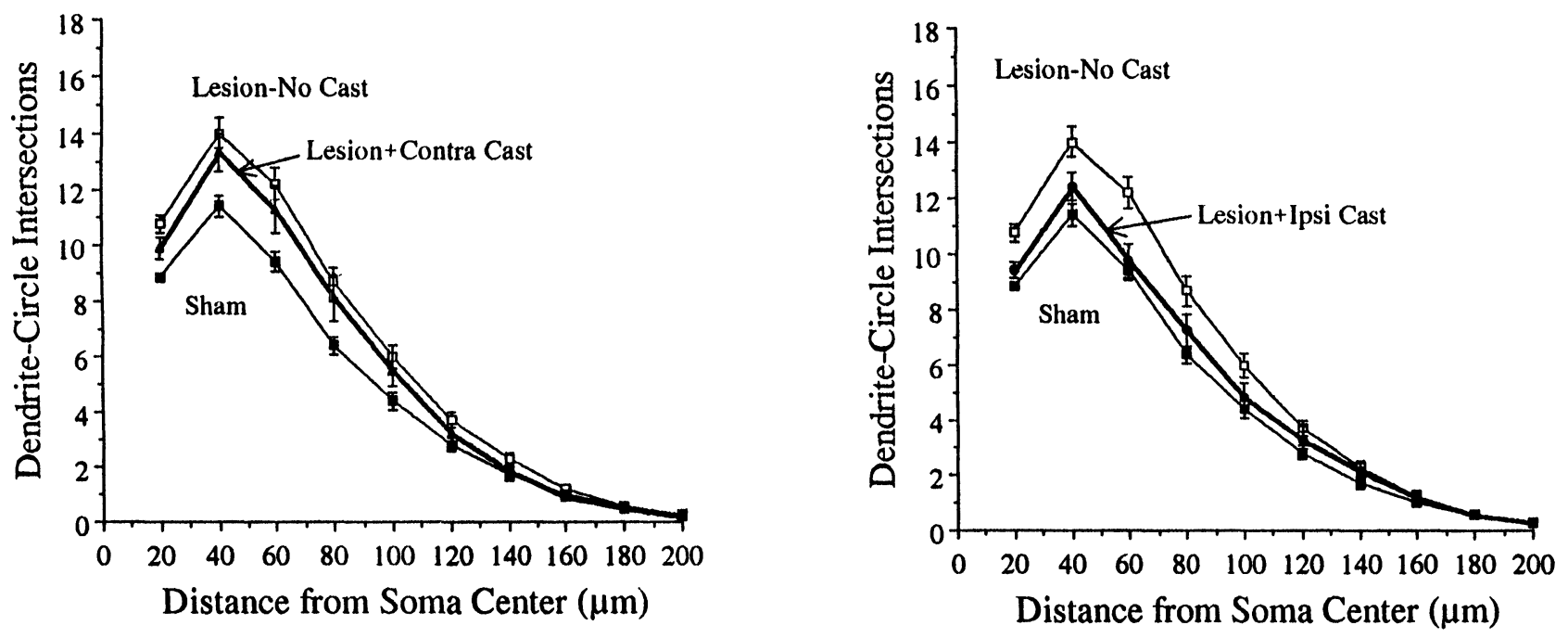

Fig. 2: Lesioned animals that were permitted to use the intact (ipsilateral) forelimb showed increased arborization at 18 days (left). Lesioned animals with movements of the ipsilateral forelimb restricted failed to show significant increases in the levels of dendritic extent and complexity. Thus, the enhanced dendritic arborization is use-dependent (see Jones and Schallert /9/).

\section{PRUNING RELATED TO EXPERIENCE}

As noted above, after the overgrowth of dendritic arbors there was a partial reduction in dendritic complexity and size (pruning). Two possibilities are that the pruning may be related to a return to more symmetrical use of the forelimbs (i.e., less reliance on the non-impaired forelimb for postural-motor behaviors), or, in contrast, to continued dependence on and improvement in the use of the non-impaired limb for postural-motor behaviors. Therefore, an experiment was carried out in which one-sleeved casts were used starting at, and throughout, the pruning phase in order to extend the period of over-reliance on the nonimpaired limb $/ 9 /$. We found that forcing animals to continue to use only the ipsilateral (nonimpaired) forelimb throughout the period of pruning did not prevent the dendritic arbor reduction. Thus, the dendritic pruning was not related to a return to more symmetrical use of the forelimbs, and might well be a fine-tuning mechanism associated with 
increased specificity of synaptic connections and continued over-reliance on the non-impaired forelimb.

\section{FUNCTIONAL OUTCOME}

A battery of behavioral tests assessed the effects of prior casting on limb use effectiveness during postural-motor behaviors in animals with sensorimotor cortex damage. These tests indicated that casting the non-impaired limb during the period of dendritic overgrowth not only blocked the arborization, but also caused severe impairment in the coordinated use of both forelimbs, which was substantially worse than that observed in uncasted animals and in animals having the contralateral (impaired) limb casted /9/. These data show that a manipulation which disenables the dendritic overgrowth can have drastic behavioral consequences, even though the manipulation forced more extensive use of the impaired forelimb. In addition, a pharmacological manipulation (NMDA antagonist administration beginning after peak dendritic arborization) that prevented the process of pruning had an adverse effect on forelimb function $15,12 /$. The potential implications of these results for restorative neurology are that there may be special early periods after brain damage during which nonaffected systems require rehabilitative training (perhaps just as much as impaired systems) in order to maximize compensatory neural mechanisms and clinical outcome.

\section{CONCLUSION}

Brain injury can create, for a short period of time, a particularly fertile milieu for compensatory structural changes that rival in scope the plasticity seen in development. We have described experiments depicting post-lesion neural morphological growth that was not unlike development-associated overproduction (exuberance) and selective elimination of neurons or their processes (pruning), a growth pattern suggested to be related to activity-dependent selective elimination of some synaptic connections and the strengthening of remaining, functionally appropriate, connections $/ 1,4,13-15,20,22,24 /$. The window of time soon after brain damage may represent a "golden" opportunity for substantial remodeling and, potentially, restoration of function. However, this opportunity may be wasted unless there is also appropriate rehabilitative experience.

\section{ACKNOWLEDGEMENT}

Supported by NIH grant NS23964 and MH18837. We thank Terry Robinson and Shari Barnes for helpful comments on the manuscript.

\section{REFERENCES}

1. Changeux JP, Danchin A. Selective stabilisation of developing synapses as a mechanism for the specification of neuronal networks. Nature 1976; 264 : 705-712.

2. Cowan WM, Fawcett JW, O'Leary DDM, Stanfield BB. Regressive events in neurogenesis. Science 1984; 225: 1258-1265.

3. Greenough WT, Larson JR, Withers GS. Effects of unilateral and bilateral training in a reaching task on dendritic branching of neurons in the rat motorsensory forelimb cortex. Behav Neural Biol 1985; 44: 301-314.

4. Hubel DH, Wiesel TN. Binocular interaction in striate cortex of kittens reared with artifical squint. J Neurophysiol 1965; 28: 1041-1059.

5. Jones TA, Kozlowski D, Schallert T. Unilateral sensorimotor cortex lesions in adult rats: enhanced dendritic arborization in the contralateral cortex is blocked by preventing use of the non-impaired limb. Soc Neurosci Abstr 1991; 17: 534.

6. Jones TA, Schallert T. Sensorimotor cortex lesions: time-dependent anatomical changes specific to the contralateral homotopic cortex. Soc Neurosci Abstr 1989; $15: 1223$.

7. Jones TA, Schallert T. Unilateral lesions to the sensorimotor cortex: behaviorally-linked dendritic changes in the contralateral cortex. Soc Neurosci Abstr 1990; 16: 44.

8. Jones TA, Schallert T. Overgrowth and pruning of dendrites in adult rats recovering from neocortical damage. Brain Res 1992; 581: 156-160.

9. Jones TA, Schallert T. Use-dependent growth of pyramidal neurons after neocortical damage. J Neurosci 1993; in press.

10. Kolb B, Gibb R. Environmental enrichment and cortical injury: behavioral and anatomical consequences of frontal cortex damage in rats. Cerebral Cortex 1991; 1: 189-198. 
11. Kolb B, Whishaw IQ. Plasticity in the neocortex: mechanisms underlying recovery from early brain damage. Prog Neurobiol 1989; 32: 235-276.

12. Kozlowski DA, Jones TA, Schallert T. Enhanced dendritic arborization and pruning after sensorimotor cortex damage in adult rats: role of the NMDA receptor. Soc Neurosci Abstr 1992; 18: 345.

13. Lo YJ, Poo MM. Activity-dependent synaptic competition in vitro: heterosynaptic suppression of developing synapses. Science 1991; 254: 1019-1022.

14. Mattson MP. Cellular signaling mechanisms common to the development and degeneration of neuroarchitecture. A review. Mech Age Dev 1989; 50: 103 157.

15. Mattson MP, Rydel RE, Lieberburg I, SmithSwintosky VL. Altered calcium signaling and neuronal injury: Stroke and Alzheimer's disease as examples. Ann NY Acad Sci 1993; 679: 1-21.

16. O'Leary DDM, Stanfield BB. A transient pyramidal tract projection from the visual cortex in the hamster and its removal by selective collateral elimination. Dev Brain Res 1986; 27: 87-99.

17. Oppenheim RW. Cell death during development of the nervous system. Ann Rev Neurosci 1991; 14: 453-501.

18. Purves D, Hume RI. The relation of postsynaptic geometry to the number of presynaptic axons that innervate autonomic ganglion cells. J Neurosci 1981; 1: 441-452.

19. Purves D, Lichtman JW. Elimination of synapses in the developing nervous system. Science 1980; 210: 153-157.
20. Purves D, Riddle DR, LaMantia AS. Iterated patterns of brain circuitry (or how the cortex gets its spots). Trends Neurosci 1986; 15: 362-368.

21. Rakic P, Bourgeois JP, Eckenhoff ME, Zecevic N, Goldman-Rakic PS. Concurrent overproduction of synapses in diverse regions of the primate cerebral cortex. Science 1986; 232: 232-235.

22. Schallert T, Whishaw IQ. Neonatal hemidecortication and bilateral cutaneous stimulation in rats. Dev Psychobiol 1985; 18 (6): 501-514.

23. Teitelbaum P, Cheng MF, Rozin P. Development of feeding parallels its recovery after hypothalamic damage. J Comp Physiol Psychol 1969; 67: 430-441.

24. Turner AM, Greenough WT. Differential rearing effects on rat visual cortex synapses. I. Synaptic and neuronal density and synapses per neuron. Brain Res 1985; 329: 195-203.

25. Whishaw IQ, Pellis SM, Gorny B, Kolb B, Tetzlaff W. Proximal and distal impairments in rat forelimb use in reaching follow unilateral pyramidal tract lesions. Behav Brain Res, in press.

26. Wiesel TN, Hubel DH. Single-cell responses in striate cortex of kittens deprived of vision in one eye. J Neurophysiol 1963; 26: 1003-1017.

27. Withers GS, Greenough WT. Reach training selectively alters dendritic branching in subpopulations of layer II-III pyramidals in rat motor-somatosensory forelimb cortex. Neuropsychologia 1989; 27: 61-69. 

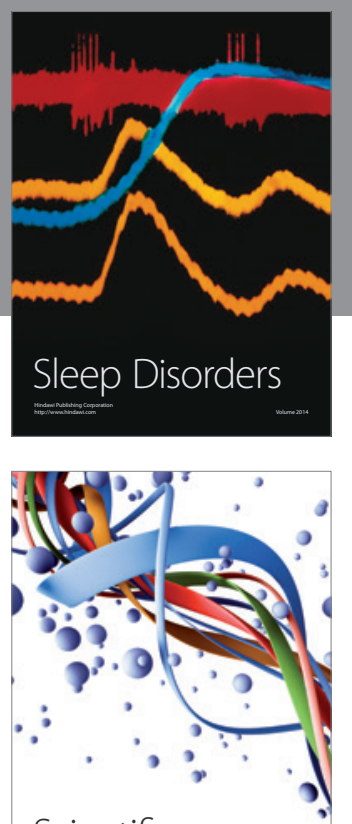

Scientifica
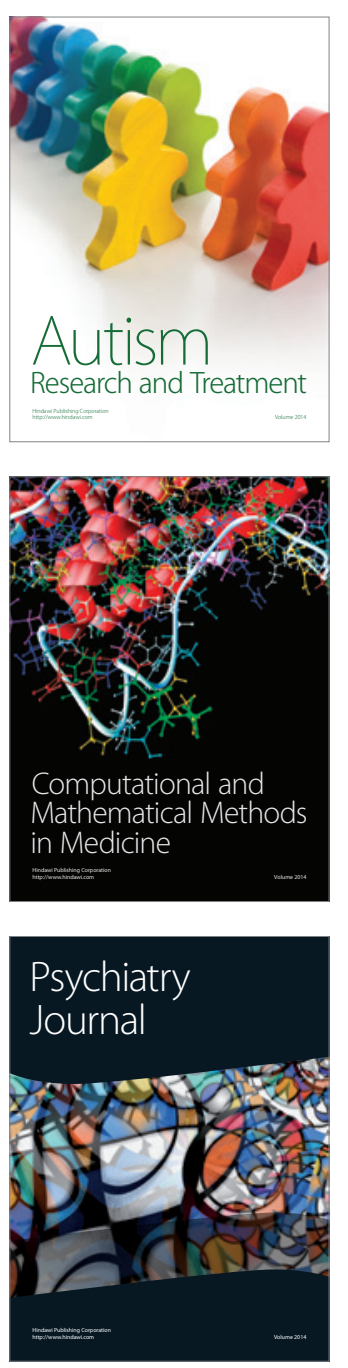
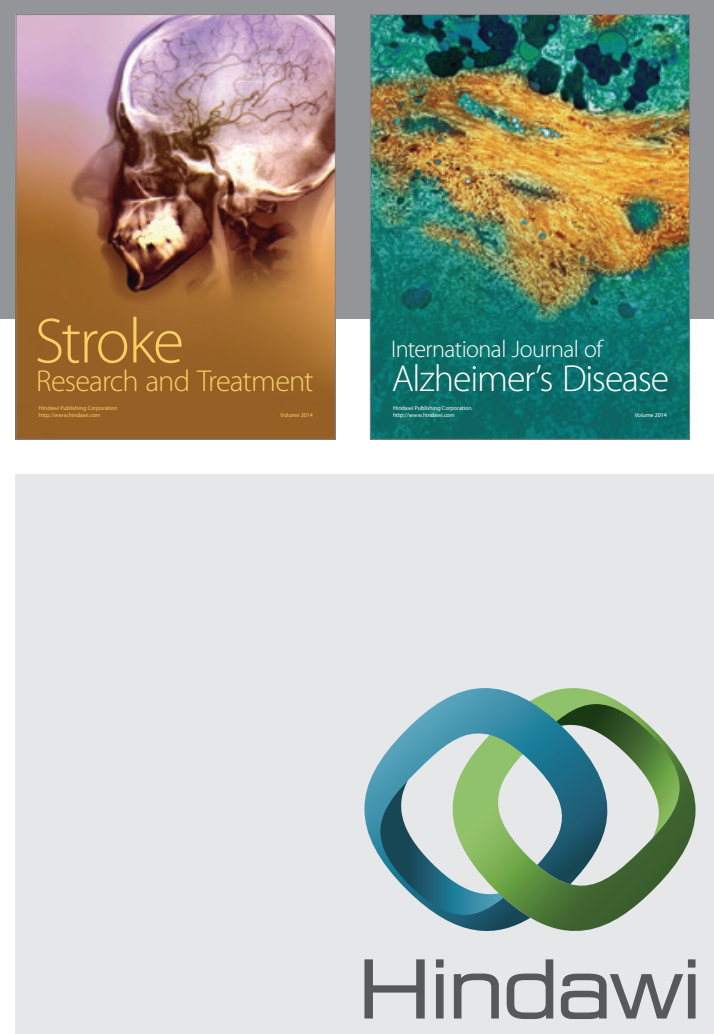

Submit your manuscripts at

http://www.hindawi.com
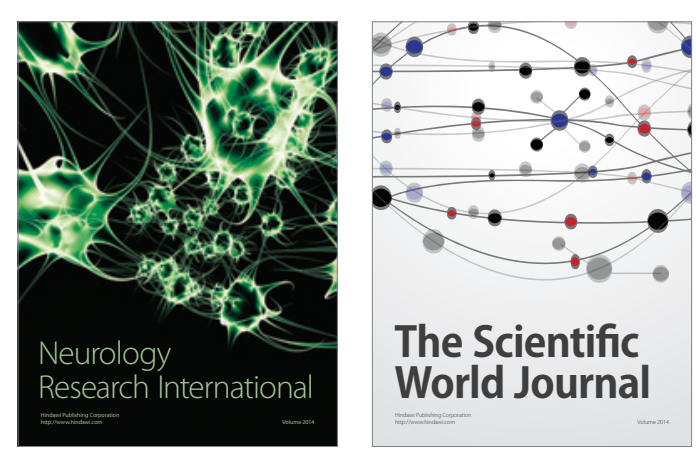

The Scientific World Journal

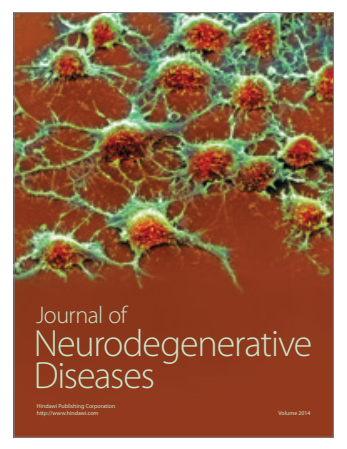

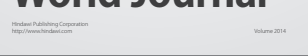

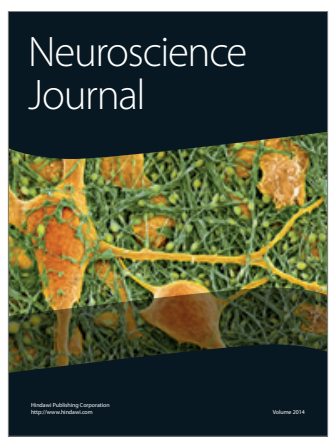

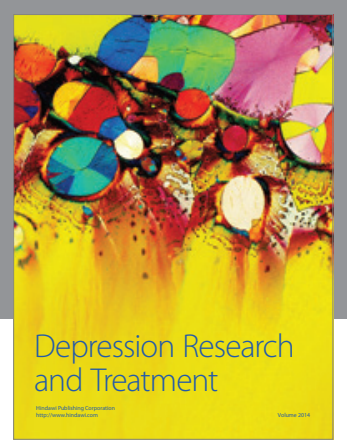
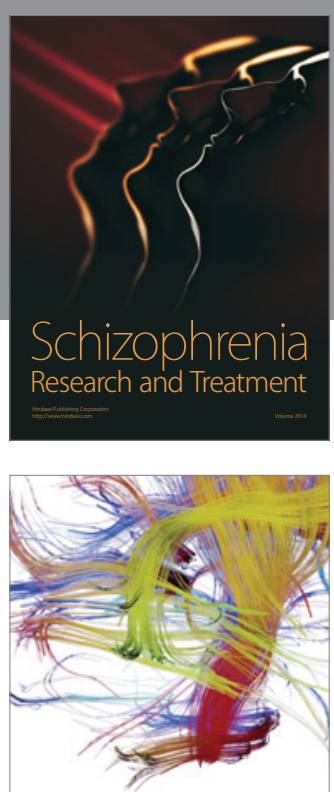

Brain Science

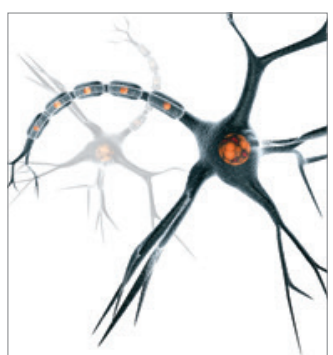

Neural Plasticity
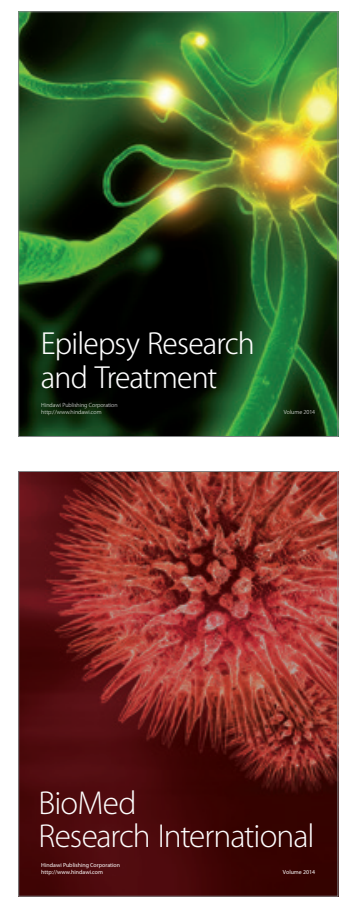

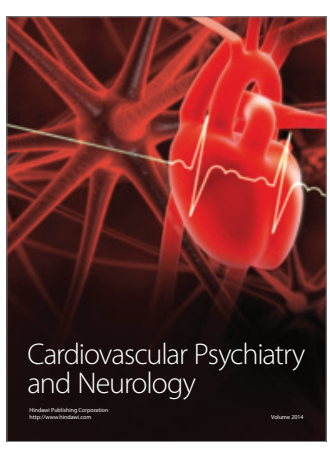

Parkinson's

Disease
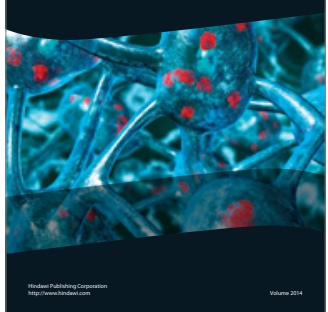